\title{
Efectividad clínica en el cuidado del orificio de salida del catéter peritoneal
}

\author{
*Antonia Gil Gil, *Elena Gisbert Rosique, **Miguel Castillo Díaz, ***María Eva Baró Salvador
}

* Enfermeras Unidad de Diálisis Hospital de Torrevieja

** Doctor en Metodología de las Ciencias del Comportamiento por la Universidad de Granada

***Nefróloga, Jefa de Servicio de Nefrología del Hospital de Torrevieja y Vinalopó (Elche)

\section{Resumen}

Existe una gran variedad de procedimientos y métodos, seguidos por los profesionales para realizar las curas y el cuidado del orificio de salida del catéter peritoneal. Averiguamos que todos ellos eran fundamentados y con resultados efectivos. Pero, nos preguntamos, ¿Cuál de entre ellos sería el más adecuado utilizar en nuestra unidad?

Optamos por utilizar las recomendaciones de la literatura científica, incluyendo variables como las necesidades específicas de cada paciente, según las condiciones medioambientales de nuestra comunidad y las estaciones del año.

El objetivo principal de nuestro estudio fue evaluar la validez y efectividad del protocolo que establecimos para nuestros pacientes.

Realizamos un estudio epidemiológico descriptivo y longitudinal de 2 años y 8 meses de duración.

Calculamos la tasa de incidencia de infecciones del orificio de salida del catéter $\mathrm{y}$ de peritonitis por paciente y para cada uno de los años de estudio (2008-20092010). Evaluamos los resultados comparándolos con

Correspondencia:

Antonia Gil Gil

Unidad de diálisis, Hosp. de Torrevieja

Ctra. Torrevieja a San Miguel de Salinas

CV-95, Partida la Ceñuela 03186

Torrevieja, Alicante los índices de normalidad marcados por la Sociedad Española de Nefrología, en el año 2007.

Desglosamos el total de infecciones según estaciones y calculamos el porcentaje. Diferenciamos entre las infecciones provocadas por bacterias gram $+y$ aquellas producidas por bacterias gram-.

Los resultados obtenidos revelaron que nuestro protocolo es válido y adecuado para nuestros pacientes, obteniendo una tasa de incidencia global de peritonitis de 0.72 por cada 24 paciente-mes y una tasa de infección del orificio de salida de 0.61 paciente-año, ambas por debajo del estándar establecido por la Sociedad Española de Nefrología (2007).

Se demostró una mayor incidencia de infecciones en el periodo estival, concretamente el $46 \%$.

La clorhexidina al $1 \%$ abarca la cobertura de las bacterias gram+, bacterias gram-, esporas, virus y hongos pero se comprueba su mayor efectividad con los microorganismos gram+.

\section{PALABRAS CLAVE:}

- CURA DEL ORIFICIO DE SALIDA

- CATÉTER PERITONEA

- CLORHEXIDINA

- INFECCIÓN DEL ORIFICIO DE SALIDA

- PERITONITIS 


\section{Clinical effectiveness in peritoneal catheter exit orifice care}

\begin{abstract}
There is a great variety of procedures and methods followed by the professionals who provide peritoneal catheter exit orifice care. We found that all of them had a sound basis and showed effective results. However, we asked ourselves which of them would be the most suitable to use in our unit.
\end{abstract}

We opted to use the recommendations of scientific literature, including variables such as the specific needs of each patient, according to the environmental conditions of our community and the seasons of the year.

The main objective of our study was to assess the validity and effectiveness of the protocol we had established for our patients.

We carried out a descriptive and longitudinal epidemiological study lasting 2 years and 8 months.

We calculated the incidence rate of catheter exit orifice infections and peritonitis per patient and for each of the years of the study (2008-2009-2010). We assessed the result by comparing them with the normality indices set by the Spanish Nephrology Association, in 2007.

We broke down the total of infections according to seasons and calculated the percentage. We differentiated between infections caused by gram + and gram - bacteria.

The results obtained showed that our protocol is valid and suitable for our patients, obtaining an overall peritonitis incidence rate of 0.72 for every 24 patient-months and an exit orifice infection rate of 0.61 patient-years, both of which are lower than the standard established by the Spanish Nephrology Association (2007).

A higher incidence of infections was found in the summer period, specifically $46 \%$.

$1 \%$ chlorhexidine covers gram + bacteria, grambacteria, spores, viruses and fungi but was found to be most effective with gram+ microorganisms.

\section{KEY WORDS:}

EXIT ORIFICE CARE

- PERITONEAL CATHETER

CHLORHEXIDINE

EXIT ORIFICE INFECTION

PERITONITIS

\section{Introducción}

El cuidado del orificio de salida del catéter peritoneal, cobra especial importancia debido a las implicaciones que producirá sobre el éxito y futuro de la diálisis peritoneal. En efecto, las infecciones del orificio de salida (0.S) provocan tratamientos prolongados, causando entre un $10-25 \%$ de peritonitis ${ }^{(1)}$, pueden ser la causa de la retirada del catéter en más de una quinta parte de los casos y son responsables de salida de la técnica en un 2 - $37 \%$ las situaciones ${ }^{(2,3)}$.

Los cuidados para prevenir la infección varían desde el lavado diario con jabón o un jabón antiséptico, hasta la cura con diferentes soluciones antisépticas o la aplicación de antibióticos. Para la apertura de nuestra unidad, comprobamos visitando otros hospitales y tras realizar una revisión bibliográfica, que existía una gran variedad de pautas a seguir, todas ellas totalmente justificadas y eficaces, en los ámbitos en las que se empleaban. No obstante, la evidencia subraya que en muchos casos las prácticas se basan en la opinión y la experiencia siendo solo en pocas ocasiones evaluadas rigurosamente ${ }^{(2)}$.

Asimismo, para la elección de las pautas a seguir, optamos en nuestra unidad, por utilizar las recomendaciones de la evidencia científica ${ }^{(2,4,5)}$, incluyendo otras variables a tomar en consideración como:

- Las necesidades específicas de cada paciente que pudieran influir en su calidad de vida y bienestar.

- Los patrones medioambientales de nuestra comunidad, según las estaciones del año.

A partir de las recomendaciones y premisas establecidas, realizamos un protocolo inicial de cura de 0.5 del 
catéter peritoneal. Asimismo, nos planteamos que ese protocolo tendría que evaluarse y reajustarse anualmente en busca de la excelencia en los cuidados.

El objeto principal de este trabajo es evaluar la validez y efectividad del protocolo establecido para los pacientes de nuestra unidad.

Los objetivos secundarios son:

Establecer un plan de seguimiento y mejora de la cura del OS del catéter peritoneal.

Averiguar si existen estaciones más propensas a la declaración de infecciones del $0 . S$ del catéter peritoneal en nuestros pacientes. (Valoración de la tasa de infecciones del $0 . S$, según el periodo estacional del año).

\section{Material y método}

Realizamos, en la Unidad de Diálisis del Hospital de Torrevieja, un estudio epidemiológico descriptivo y longitudinal de una duración de 2 años y 8 meses.

La enfermería basada en la evidencia $(E B E)$ es el método que nos permitió, de forma juiciosa y explícita, obtener con la utilización de la documentación científica, las mejores pruebas disponibles para el cuidado del $0 . S$ en nuestros pacientes. Entre las bases de datos que fueron consultadas destacan medline, Cochrane, cuiden, Pubmed, cuidatge.

\section{Evidencias disponibles}

\section{- Aspectos generales para prevenir infecciones:} Se destaca la fijación del catéter, la higiene, la atención meticulosa, la importancia del lavado de manos y el secado riguroso (2).

\section{- Antibióticos:}

En el grupo de fármacos con uso profiláctico para la prevención de infección de 0 .S se encuentra la mupirocina, el ciprofloxacino y la gentamicina ${ }^{(2,4,6)}$.

No existen suficientes estudios que nos permitan conocer la resistencia antibiótica en periodos largos ${ }^{(2)}$, aun así en algunos estudios se han informado de casos de resistencia a la mupirocina cuando se usa de manera intermitente ${ }^{(6)}$.

\section{- Antisépticos:}

La povidona yodada y la el gluconato de clorhexidina entre el $0.05-1 \%$ se consideran opciones de tratamiento razonables. Por otra parte se indica que se debe de evitar el uso diario de peróxido de hidrógeno por su acción secante ${ }^{(6)}$.

\section{- Apósitos:}

Según la evidencia existente, ninguna técnica sobre apósitos puede ser altamente recomendada como más efectiva que otra para la reducción o prevención de infecciones ${ }^{(2)}$.

\section{Recomendaciones de las casas comerciales sobre los antisépticos a utilizar para la cura de sus catéteres}

Se recogieron las recomendaciones de las casas comerciales sobre los antisépticos y antibióticos aconsejados y aplicables al catéter, en el momento de la cura.

\section{- Catéteres de silicona:}

casas comerciales aconsejan la povidona iodada de base acuosa como desinfectante de elección. Antiséptico contraindicado: desaconsejan la utilización de acetona o alcohol y por tanto toda solución que los contenga.

\section{- Catéteres de poliuretano:}

Antibiótico de uso tópico autorizado: ciprofloxacino. Antibióticos de uso tópico y antisépticos contraindicados: se descarta el uso de povidona yodada y mupirocina.

\section{Diseño del protocolo de cura de orificio de salida}

El protocolo de cura del $0 . S$ se realizó a partir de la evidencia disponible y fue aprobado por los responsables de la unidad. (figura 1 )

\section{Procedimiento de cura del orificio de salida del catéter de peritoneal:}

\section{Post operatorio:}

El 0.S durante el periodo de cicatrización es curado, mediante técnica estéril, una vez a la semana por el 
personal de la unidad de diálisis peritoneal. Se realizan las curas con limpieza de suero fisiológico, secado minucioso y aplicación de clorhexidina $1 \%$ hasta cica- trización. El periodo de cicatrización comprende las primeras 4-6 semanas tras la implantación del catéter, dependiendo de la evolución de cada orificio.

\begin{tabular}{|c|c|c|c|c|}
\hline $\begin{array}{c}\text { Clasifición } \\
\text { twardowski }\end{array}$ & Agua y jabón & Apósito & Antiséptico & $\begin{array}{c}\text { Antibiótico } \\
\text { preventivo }\end{array}$ \\
\hline 0. Perfecto & SI & preferencia paciente & \\
\hline 0. Bueno & SI & SI & SI & \\
\hline 0. Equívoco & SI & SI & & SI \\
\hline 0. Infección crónica & SI & SI & \\
\hline 0. infección aguda & SI & SI & & SI \\
\hline 0. Traumático & SI & SI & & S valorar \\
\hline
\end{tabular}

Figura 1. Protocolo de cura del OS

\section{Paciente:}

Tras la cicatrización los pacientes entrenados, realizan el autocuidado y cura de su O.S.

La técnica a seguir incluye lavado de orificio con agua y jabón neutro, secado minucioso, aplicación de antiséptico y apósito según necesidades y preferencias del paciente. El antiséptico de elección es la clorhexidina $1 \%$ de base acuosa. Este procedimiento se realizará hasta la completa maduración del orificio como mínimo durante seis meses. Una vez transcurrido este tiempo, si el orificio es clasificado en el estadio de perfecto se procederá a realizar cura sólo con agua y jabón con secado minucioso y apósito si requiere.

\section{Valoración del orificio de salida}

La valoración del estado del $0 . S$ se realizó cada 2 meses a partir de la tabla de clasificación histomorfológica de Twardowski. Se realizó también, una recogida de muestras para cultivo del orificio de salida cada 6 meses de forma estandarizada. Se amplió el número de valoraciones y cultivos realizados, según indicación médica, ante la sospecha de posible infección o como seguimiento de la infección ya establecida.

El criterio que se siguió para reconocer un episodio de infección fue: incluir todos los 0.5 catalogados como equívocos, con inflamación crónica o inflamación aguda y que además presentasen un cultivo positivo en ese momento.

El diagnóstico fue realizado por un único observador que realizó todas las evaluaciones a lo largo del estudio.

\section{Datos recogidos en el estudio}

Para el registro de la información se creó una base de datos en formato Excel 2007 diseñada específicamente para el estudio. En esta se incluyeron, para cada paciente, los siguientes parámetros: fecha de colocación del catéter peritoneal, fecha de retirada del catéter, número de días expuesto a riesgo de infección, número de peritonitis, número de infecciones del orificio de salida, fecha en la que se ha producido la infección y tipo de microorganismo causal.

\section{Método de valoración de los resultados}

Con la finalidad de conocer la efectividad de nuestro protocolo en cada uno de los años se calculó la tasa de incidencia (densidad de incidencia) de las infecciones del orificio de salida y la tasa de incidencia de peritonitis por paciente y año. Los resultados se compararon con los índices de normalidad marcados en el plan de calidad científico técnica y de mejora continua de calidad 
en diálisis peritoneal establecido por la SEN en el año 2007. (Menos de 1 episodio cada 24 paciente-meses en el caso de la peritonitis y entre 0.05 y 1.02. episodios/ paciente-año en caso de infección del 0.S ${ }^{(1)}$. La tasa de incidencia indica el número de sujetos que pasan de sanos a enfermos por unidad de tiempo en relación al tamaño de la población en riesgo. Mide el número de casos que se producen por unidad de tiempo y población susceptible, o lo que lo mismo, la fuerza de morbilidad de la enfermedad en dicha comunidad. La tasa de incidencia se calcula dividiendo el número de episodios nuevos de enfermedad durante el periodo de seguimiento entre la sumatoria de los periodos individuales en riesgo. Para su cálculo medimos el tiempo que cada paciente estuvo en riesgo, lo que se conoce como periodo individual en riesgo. Este periodo es igual al tiempo que transcurre desde que un paciente susceptible de presentar la enfermedad comienza a ser observado hasta que deja de estar en riesgo, ya sea porque sufre la enfermedad, porque muere, porque se pierde el seguimiento o porque sale de la situación en riesgo. El tiempo de la tasa de incidencia por estar referido a personas se expresa en unidades de persona-tiempo.

Consideramos que el paciente estaba expuesto a riesgo desde que se colocó el catéter y dejaba de estar en riesgo al darse de baja.

La fórmula que aplicamos fue la siguiente: Tasa de Incidencia $=\mathrm{N}^{\circ}$ de Inicios nuevos de Enfermedad / Suma periodos individuales de tiempo en riesgo

Por otra parte, se desglosó el total de infecciones en base el periodo estacional en el que se produjo, calculando el porcentaje en cada caso. Asimismo, se diferenció entre aquellas infecciones provocadas por bacterias gram + y aquellas producidas por bacterias gram-.

Realizamos un contraste estadístico para proporciones para comprobar si la tasa obtenida difería significativamente del estándar.

\section{Tamaño muestral}

El tamaño total de la muestra fue de 20 pacientes, de los cuales 10 fueron hombres y 10 mujeres, con edades comprendidas entre 34 y 80 años. Se incluyó a todos los pacientes portadores de catéter peritoneal de nuestra unidad. Admitimos que la entrada de los pacientes fuera progresiva a lo largo del periodo de estudio.
Cronológicamente obtuvimos una muestra de: 5 personas en el 2008, de 12 personas en el año 2009 y de 17 personas en el 2009.

\section{Resultados}

Con el fin de conocer la eficacia de nuestro protocolo, se realizó una evaluación global de los resultados.

Se analizó de forma específica la tasa de incidencia de infecciones de 0.S en cada uno de los años del periodo. Se observa que en el año 2008 la tasa de infecciones es de 1.90 episodios/paciente año, en el año 2009 la tasa es de 0.70 episodios/paciente - año, $Z=-4.63, p<.001$, y en el año 2010 la tasa es de $0.39, Z=-11.13, p<.001$. Todos los años, a excepción de 2008, ofrecen valores dentro del estándar establecido por la SEN (2007). Realizamos un cálculo de la tasa de incidencia de infecciones de 0.S. para todo el periodo de tiempo del estudio, obteniendo una tasa de 0.61 episodios/paciente-año, $Z=-7.45, p<.001$. De igual forma, esta tasa es significativamente menor que el estándar establecido por la SEN (2007).

Durante el año 2008 y 2009 se dieron un total de 8 episodios de infección de orificio de salida, de los cuales, 4 de ellos ocurrieron justo después de suspender el uso de clorhexidina al $1 \%$ y pasar a usar solamente agua y jabón. A partir del 2009 se decide curar siempre el $0 . S$ con agua y jabón seguido de un secado minucioso y aplicación posterior de clorhexidina.

En la figura 3, observamos la evolución de la tasa de infecciones de 0.S en nuestra unidad. Vemos que esta tasa se reduce considerablemente a través del tiempo, acercándose a una mayor estabilidad a medida que han aumentado el número de pacientes.

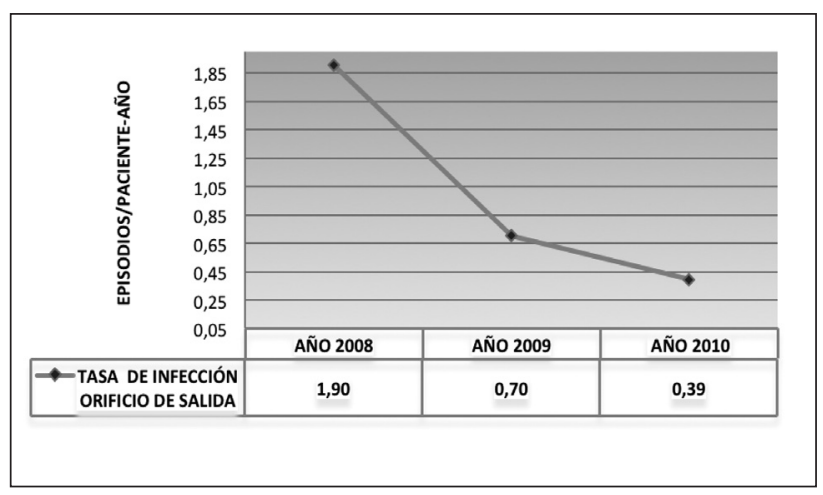

Figura 3. Infección orificio de salida 
Los episodios de infección que se han producido a lo largo de todo el periodo de estudio han sido agrupados según la estación del año en que ha tenido lugar. Se observa que el mayor índice de infección se encuentra en el periodo estival (46,15\%). (figuras 2 y 4 ).

\begin{tabular}{|c|c|c|c|c|}
\hline VARIABLES MEDIDAS & $\begin{array}{c}\text { AÑO } 2008 \\
n=5\end{array}$ & $\begin{array}{c}\text { AÑO } 2009 \\
n=12\end{array}$ & $\begin{array}{c}\text { AÑO } 2010 \\
n=17\end{array}$ & $\begin{array}{c}\text { TOTAL PERIODO } \\
n=20\end{array}$ \\
\hline RATIO DE PERITONITIS & 0 & 0,82 & 0,46 & 0,72 \\
\hline RATIO DE INFECCION O.S & 1,90 & 0,70 & 0,39 & 0,61 \\
\hline$\%$ INFECCION M.O GRAM + & 66,67 & 40 & 20 & 38,46 \\
\hline \% INFECCION M.O GRAM - & 33,33 & 60 & 80 & 61,54 \\
\hline \multirow{4}{*}{$\begin{array}{l}\text { ESTACIONES } \\
\% \text { infecciones }\end{array}$} & PRIMAVERA & 0 & 20 & 0 \\
\hline & VERANO & 66,67 & 40 & 40 \\
\hline & OTOÑO & 33,33 & 20 & 20 \\
\hline & INVIERNO & 0 & 20 & 40 \\
\hline
\end{tabular}

Figura 2. Tasas de infecciones según estaciones

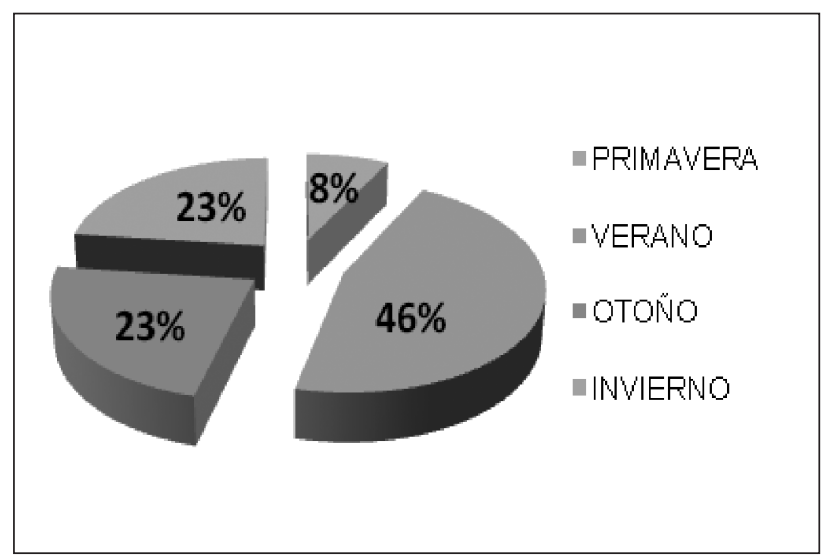

Figura 4. Proporción de infecciones según estaciones, años 2008. 2009. 2010

Partiendo del total de infecciones registradas durante 2 años y 8 meses (13 episodios), evidenciamos las infecciones por microorganismos (m.o) gram-abarcaban un $61,54 \%$ y las infecciones por m.o gram+ el $38.46 \%$ (figura 5 ).

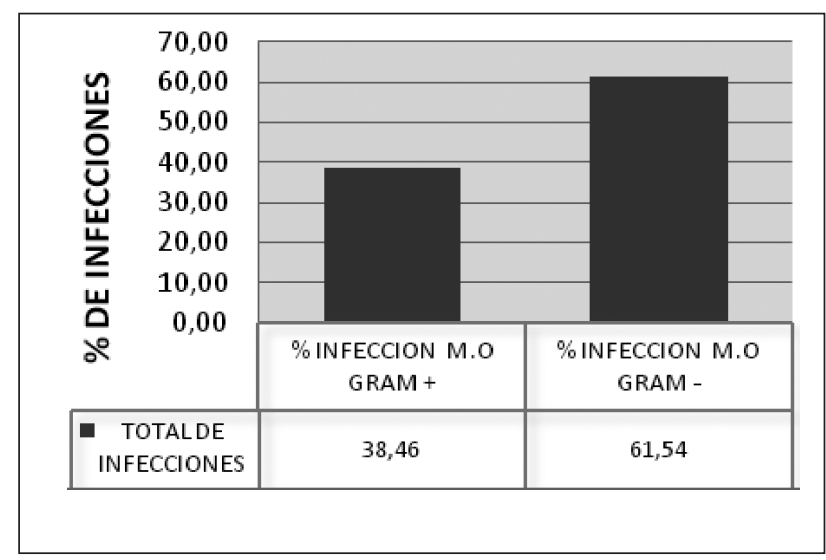

Figura 5. Clasificación de infecciones

Para finalizar se comprobaron la tasa de peritonitis en cada año de estudio. Tal y como se aparecen en la Figura 6, las tasas de incidencia de peritonitis fueron menor a 1 episodio por cada 24 paciente-mes. El contraste estadístico de proporciones para comprobar si la 
tasa obtenida difería significativamente del estándar demuestra que tanto para el año 2009, $Z=-2.71, p=$ .003 , como para el año 2010, $Z=-9.86, p<.001$, como para el periodo total de estudio, $Z=-5.20, p<.001$, la tasa de incidencia de peritonitis ha sido significativamente menor que el estándar establecido. Además en ningún caso estos episodios estuvieron asociados a infección del orificio de salida.

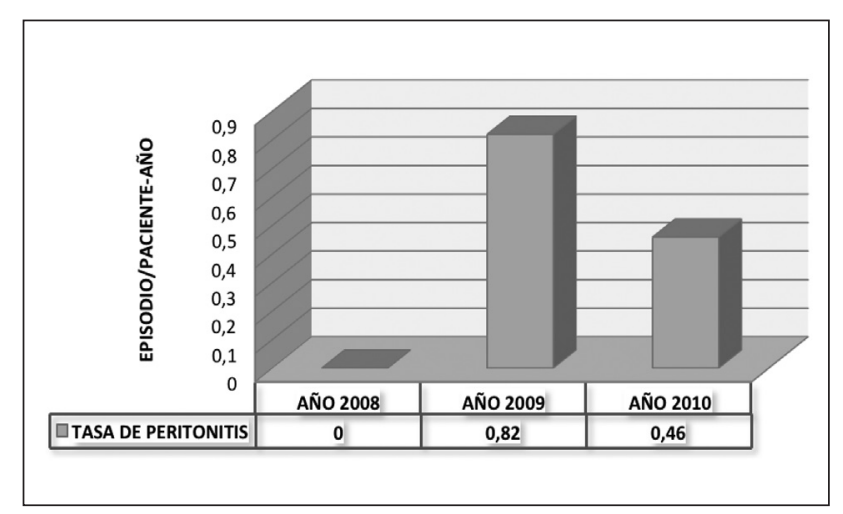

Figura 6. Tasa de peritonitis por paciente y año

\section{Discusión}

Según indica la SEN (2007), en el plan de calidad científico técnica y de mejora continua de calidad en diálisis peritoneal, las tasas de infección del catéter descritas son muy variables: entre 0.05 y 1.02 episodios/paciente-año. Por otra parte, se evidencia que con aplicación continua de antibióticos en el orificio del catéter se consiguen tasas de 0.06 episodios/ paciente-año con ciprofloxacino ótico en el orificio y de 0.54 episodios/paciente-año con mupirocina vs. 0.23 episodios/paciente-año con gentamicina crema local.

En nuestra unidad, tras el periodo de seguimiento de los 0.S, el resultado obtenido ha sido de 0.61 episodios/ paciente-año. Se ha observado un descenso paulatino de las infecciones siendo en el último año de 0.39. En el año 2008 la tasa de infecciones, 1.90 episodios/ paciente -año, es mayor al standard. Es importante incidir que el número de pacientes en el año 2008 fue muy reducido, sólo 5 personas, haciendo que la ratio obtenida sea poco representativa y muy elevada para sólo 4 episodios de infecciones en todo el año. Por último, hay un claro descenso del número de infecciones de orificio de salida a lo largo del tiempo.
Los dos primeros años observamos que tras suspender el uso de la clorhexidina al $1 \%$ tuvimos un incremento de los episodios infecciosos, por lo que se decidió reintegrar de nuevo el uso de este antiséptico a partir de 2009.

La tasa de peritonitis en cada uno de los años de estudio ha estado por debajo del estándar fijado.

Estos resultados nos llevan a concluir que el protocolo de cura de orificio de salida, que actualmente seguimos en nuestra unidad, es válido y adecuado para nuestros pacientes.

Por otra parte, los resultados del estudio muestran una mayor incidencia de infecciones en el periodo estival. Consideramos, por lo tanto la necesidad de aumentar nuestros niveles de alerta en ese periodo y valorar la posibilidad de utilizar, para los pacientes con mayor riesgo, un antibiótico de manera profiláctica.

La clorhexidina al $1 \%$ es considerada como un antiséptico de rápida actuación y además de amplio espectro de acción. La protección de este antiséptico abarca la cobertura de las bacterias gram +, bacterias gram-, esporas, virus y hongos. Así mismo es de resaltar que la literatura destaca una mayor efectividad de la clorhexidina con los gram+. Nuestros resultados se encuentran en concordancia con esta afirmación.

Por último, queremos apuntar algunas limitaciones del estudio que pueden disminuir la potencia del efecto encontrado. En primer lugar, la muestra es pequeña llevándonos a ser cautos en las tasas de incidencia calculadas y en los contrastes estadísticos aplicados a las tasas de peritonitis. En segundo lugar, se calcularon porcentajes para las infecciones en función de la estación del año y en función del tipo de bacteria con un $\mathrm{N}<20$. De esta forma, se propone seguir la línea de investigación con un estudio con carácter confirmatorio aumentando la muestra y la duración del estudio longitudinal.

La bibliografía indica que el uso de antibióticos de forma rutinaria podría fomentar aún más la disminución de nuestra tasa de infecciones $(0,38)$, no obstante la falta de estudios sobre las resistencias alcanzadas en la aplicación sistemática de un antibiótico nos ha dirigido a considerar su uso tan solo cuando existía infección o un riesgo real de padecerla. 
No podemos descartar que la experiencia de la responsable de la unidad de peritoneal y la buena predisposición de nuestros pacientes en aplicar el protocolo pueda haber influido en los buenos resultados obtenidos.

Todo el estudio fue controlado y procesado por una única profesional la cual puso especial atención en evitar alteraciones en la aplicación del protocolo. Así la mejoría de los resultados puede atribuirse también a la profilaxis aplicada en todas las fases del protocolo.

Por otra parte, es preciso resaltar el carácter exploratorio de esta investigación proponiendo nuevas líneas de estudio en las que, por ejemplo, se evalúe también la efectividad de nuestro protocolo en base a otras variables como por ejemplo el tipo de actividad de nuestros pacientes (laboral, ocio, sedentarismo), considerando cualquier actividad o inactividad puede generar riesgos añadidos en el mantenimiento del cuidado del orificio del catéter. Para terminar, notificar que nos hemos planteado el uso de un antiséptico distinto de la clorhexidina para intentar paliar los episodios repetitivos de infecciones producidas por gram - en un mismo paciente.

Recibido: 10 diciembre 2011

Revisado: 6 Febrero 2012

Modificado: 28 Mayo 2012

Aceptado: 2 Julio 2012

\section{Bibliografía}

1. Bajo $M^{a}$ Auxiliadora, Selgas Rafael: Plan de calidad científico técnica y de mejora. Sociedad Española de Nefrología (Grupo de Promoción del Conocimiento en DP), 2007.

2. The Joanna Briggs Institute: Clinical effectiveness of different approaches to peritoneal dialysis catheter exit-site care. Evidence Based Practice Information Sheets for Health Professionals, Bestpractice. 2004; 8(1): 1-7, ISSN 1329 - 1874.

3. Gupta B, Bernardini J, Piraino B: Peritonitis associated with exit-site and tunnel infections. Am J Kidney Dis 1996; 28: 415-9.

4. Del Peso G, Bajo M.A, Selgas R: Diálisis peritoneal basada en la evidencia. Nefrología. 2006; 26(5): 56-65.

5. Arrieta J, Bajo M.A, Carravaca F.: Guías de Prácticas Clinica en Diálisis Peritoneal . Sociedad Española de Nefrología 23 Octubre 2005.

6. Piraino B, Bailie G, Bernardini J, Boeschoten E, Gupta A, Clifford: Recomendaciones para las infecciones relacionadas con diálisis. Peritoneal Dialysis International. 2005; 25: 107-131. 